\section{LA PERSONALIZACIÓN DEL APRENDIZAJE: UN OBJETIVO DEL PARADIGMA EDUCATIVO CENTRADO EN EL APRENDIZAJE}

\author{
Dolores Lerís y María Luisa Sein-Echaluce \\ Escuela de Ingeniería y Arquitectura, Universidad de Zaragoza, \\ Campus Rio Ebro, \\ María de Luna 3, 50018-Zaragoza (España) \\ dleris@unizar.es,mlsein@unizar.es
}

\begin{abstract}
In this paper we present a panoramic view about the personalization of the learning process, and about the technological tools that help to carry it out. We offer a quick overview of the characteristics taken into account in the user models of the adaptive learning systems. Through our experiences, we show our contribution to the design and experimentation of adaptive learning, highlighting both the adaptation variables and the characteristics of the implemented designs.
\end{abstract}

KEY WORDS: Distance learning; adaptive learning; instructional design; LMS; Moodle.

\section{INTRODUCCIÓN}

Como señalaba David Oakley, en 1983, la transmisión del conocimiento a las siguientes generaciones es una característica de los seres humanos. A lo que Viñes (2008) añade: "Este hecho... nos hace seres en permanente aprendizaje... No podemos evitar aprender. Aprendemos de manera consciente, inconsciente, formal e informal...". En este trabajo nos referiremos al "aprendizaje intencional, al que ha sido diseñado, planificado y ejecutado con un método instruccional que busca resultados concretos" como define Viñes (2008).

Es evidente que realizar diseños instruccionales es la tarea que abordamos de forma continua y, casi rutinaria, los formadores. Un diseño instruccional, como dice Colón (2011), "es un proceso sistemático mediante el cual se analizan las necesidades y metas de la enseñanza y a partir de ese análisis se seleccionan y desarrollan las actividades y recursos para alcanzar esas metas, así como los procedimientos para

\section{THE PERSONALIZATION OF LEARNING: AN OBJECTIVE OF THE LEARNING-BASED EDUCATIONAL PARADIGM}

RESUMEN: En este artículo se presenta una panorámica general sobre la personalización del aprendizaje y sobre las herramientas tecnológicas que contribuyen a llevarla a cabo. Se da una rápida visión de las características consideradas en los modelos de usuario de los sistemas de aprendizaje adaptativo. A través de las experiencias realizadas, se muestra nuestra contribución en el diseño y experimentación de aprendizaje adaptativo, deteniéndonos especialmente en las variables de adaptación y en las características de los diseños implementados.

PALABRAS CLAVE: Aprendizaje a distancia; aprendizaje adaptativo; diseño instruccional; LMS; Moodle.

evaluar el aprendizaje en los alumnos y para revisar toda la instrucción". En la práctica, disponemos de modelos, a veces tendencias, que nos guían en la construcción de un diseño instruccional. En ese sentido, el módulo formativo de Williams, Schrum, Sangra y Guardia (2004) anuncia la existencia de muchos modelos de procesos de diseño instruccional. Así mismo, indica varios elementos básicos comunes a la mayoría de ellos, conocidos en inglés como ADDIE (acrónimo de los cinco elementos básicos: Analysis, Design, Development, Implementation y Evaluation). El primer elemento básico, Analysis, consiste en analizar a los estudiantes, al público al que va dirigida la formación. A veces ese análisis se centra en conocer los rasgos generales del público objetivo y en actuar en consecuencia, adaptando la instrucción a esos rasgos.

Ahora bien, entendemos que el paradigma educativo centrado en el aprendizaje es más ambicioso en el análisis de los estudiantes y señala, como objetivo ideal, el conocer y adaptar el aprendizaje a los rasgos de cada individuo, no 
a las características que se le presuponen como miembro de un grupo. En nuestra opinión, ya Ausubel (1978) señala la importancia del individuo cuando dice: "el factor más importante que influye en el aprendizaje es lo que el alumno sabe. Averígüese esto y enséñese en consecuencia". Es en ese contexto donde la personalización del aprendizaje constituye, desde hace algún tiempo y de forma incuestionable, una parte sustantiva de los nuevos modelos de aprendizaje e innovación en la práctica instruccional. Aunque en realidad no es un aspecto novedoso, sí nos parece que la personalización de la educación ha ido creciendo en importancia.

El interés por hacerla resurgir en estos últimos años queda patente por la ingente cantidad de congresos y publicaciones sobre el tema. Destacamos la celebración de la conferencia internacional "Personalised Learning: the Future of Public Service Reform" (Enseñanza personalizada: el futuro de la reforma de la función pública) en Londres, en mayo de 2004. El informe publicado por la OCDE, en 2006, con el título "La personalización de la enseñanza" recoge las contribuciones más importantes de dicha conferencia. Es muy interesante constatar que todas las opiniones ahi reunidas demuestran, desde diferentes puntos de vista, lo importante que sería aplicar la personalización del aprendizaje, a la vez que recogen su concepción social, organizativa e instruccional de la personalización. Podemos decir, sin lugar a dudas, que la conferencia de Londres y el informe subsiguiente de la OCDE no hacen sino plasmar un ideal: personalizar la educación. Surge inmediatamente la necesidad de descender a lo real, de describir y poner en práctica modelos de aprendizaje en los que la personalización sea un factor determinante del diseño de la instrucción.

Pero descender a lo real no es fácil. La experiencia nos ha permitido detectar algunos obstáculos que amenazan la puesta en práctica de la personalización del aprendizaje. Tradicionalmente la eficacia de la actividad del profesorado se mide por las tasas de éxito y de rendimiento, es decir, prima únicamente el grupo, no los logros de cada estudiante como individuo. A ello se añade que nuestras prácticas educativas habituales piden un ritmo uniforme de aprendizaje al grupo de estudiantes: romper esa inercia es imprescindible. Otra amenaza que ha salido a la luz es la complejidad de los diseños de la instrucción, pues es necesario establecer hitos en el proceso de aprendizaje, diseñar diferentes itinerarios, diversificar los recursos y las actividades, etc.

Afortunadamente, los avances de la tecnología de e-learning y de tratamiento de la información hacen que cada vez sea más viable aplicar la personalización del aprendizaje.

Una de las líneas de desarrollo de la tecnología e-learning, iniciada hace al menos veinte años, consiste en la creación y mejora de sistemas hipermedia adaptativos (en adelante AHS, acrónimo en inglés de Adaptive Hypermedia Systems). Estos sistemas ofrecen contenidos a sus usuarios de forma personalizada, es decir, están dotados de la capacidad de adaptar los contenidos a las características de cada usuario. La aplicación de los AHS que aquí nos interesa se refiere al aprendizaje adaptativo, los sistemas que lo soportan se suelen Ilamar Sistemas de Aprendizaje Adaptativo o Sistemas de Tutorización Inteligente. Dicho de forma muy concisa, el objetivo de tales sistemas es incorporar la tutoría personal de la enseñanza presencial al aprendizaje electrónico.

Por otro lado, los sistemas de gestión del aprendizaje (en adelante, LMS, acrónimo de Learning Management Syste$\mathrm{ms}$ ) se han desarrollado de tal manera que ofrecen entornos de aprendizaje con un amplio abanico de herramientas de aprendizaje, con facilidades para la gestión y seguimiento de usuarios y para la administración de cursos.

Ambas líneas de desarrollo (AHS y LMS) han ido convergiendo, y siguen haciéndolo, de modo que los LMS más populares tienden a integrar la posibilidad de personalizar el aprendizaje. La plataforma de e-learning Moodle (acrónimo de Modular Object-Oriented Dynamic Learning Environment), de código abierto, no ha sido ajena a esa idea. Los desarrolladores que trabajan en la mejora y avance de Moodle han ido ofreciendo, a lo largo de estos últimos años, complementos que facilitan la personalización del aprendizaje en función de las características del estudiante.

Tras algunos intentos de añadir adaptatividad a Moodle, bien en algunas actividades, como la Lección, o bien a través de módulos externos, como Activity Locking o Score Lock, la última versión de Moodle (2.1) integra la posibilidad de bloquear una actividad en tanto no se cumplan los criterios establecidos por el profesor en su configuración. 
En esa línea, el Centro de Innovación para la Sociedad de la Información (en adelante, CICEI) de la Universidad de Las Palmas de Gran Canaria ha desarrollado los Ilamados Condicionales del CICEI para Moodle, desarrollo que se encuentra disponible para versiones 1.9.x (más información en CICEI (2005)). Su capacidad de personalización de aprendizaje es superior a la integrada en la versión 2.1 de Moodle. Mencionemos, a modo de ejemplo, dos aspectos importantes. Uno de ellos se refiere a la forma de conectar las condiciones o criterios de visibilidad. En efecto, Moodle 2.1 permite el conector AND, en tanto que los Condicionales del CICEI admiten, además, los restantes operadores lógicos (OR y NOT) para construir el condicional. Otra ventaja crucial radica en que los Condicionales del CICEI para Moodle permiten basar la presentación de los contenidos en algo más que el nivel de conocimiento de un estudiante. De hecho, estos condicionales nos han permitido, como exponemos más adelante, supeditar el itinerario del estudiante a sus fortalezas o debilidades en un concepto específico, dependiendo de la opción seleccionada por el estudiante en una determinada pregunta.

Las sucesivas experiencias de personalización del aprendizaje que hemos puesto en práctica han ido utilizando, cada vez de forma más sofisticada, la plataforma Moodle, inicialmente con el módulo externo Activity Locking de la versión 1.8, continuando con la funcionalidad Agrupamientos añadida en la versión 1.9, que permite asignar recursos y actividades a grupos de alumnos, hasta llegar a los Condicionales del CICEI para Moodle 1.9.

\section{Modelo de usuario en el aprendizaje ADAPTATIVO}

Uno de los componentes que contemplan los sistemas de aprendizaje adaptativo, que es el que aquí nos interesa destacar, es el llamado modelo de usuario. Con él se pretende recoger y estructurar las variables de personalización del aprendizaje.

Inicialmente el modelo de usuario recogía las variables que se denominan características del usuario. De hecho, Brusilovsky (1996) define un AHS como "cualquier sistema hipertexto o hipermedia que refleja algunas características del usuario en un modelo y que aplica este modelo para adaptar varios aspectos visibles del sistema para el usuario".

Las variables consideradas tradicionalmente como características del usuario han sido: nivel de conocimientos, estilo de aprendizaje y metas e intereses del usuario. Señalemos que, en el caso del nivel de conocimientos, su determinación es un área de extenso estudio en la que se utiliza el llamado Test Adaptativo Informatizado (TAI), traducción de la expresión inglesa "Computerized Adaptive Test" (CAT). Como dicen Olea y Ponsoda (2002): "... la característica fundamental de un TAl es que se va adaptando al nivel de competencia progresivo que va manifestando la persona". EI TAI mide el nivel alcanzado en cada rasgo y este es el valor usado para adaptar el programa de instrucción a esa característica del usuario.

Más adelante, el mismo Brusilovsky (2001) recoge una respuesta más amplia a la pregunta ¿adaptarse a qué? y dice: "Tradicionalmente la decisión de adaptación en los sistemas adaptativos se basaba en tener en cuenta diversas características de sus usuarios recogidas en el modelo de usuario. Eso era asi en los sistemas hipermedia adaptativos anteriores a 1996 y la respuesta a la pregunta ¿adaptarse a qué? contemplaba exclusivamente las características del usuario. En la actualidad (se refiere a 2001) la situación es diferente..... Kolba et al. (1999) sugieren distinguir entre la adaptación a los datos del usuario, a los datos de uso y a los datos del entorno." Brusilovsky sigue explicando que el modelo de usuario propuesto por Kolba es una clasificación de las variables de personalización en tres categorias:

- Los datos del usuario o lo que antes hemos llamado características del usuario.

- Los datos de uso que reúne los datos de la interacción del usuario con el sistema.

- Los datos del entorno en el que se engloban todos los datos del entorno del usuario diferentes a sus características personales.

Este mismo modelo de usuario es el propugnado por Vélez y Fabregat (2007) como un modelo integral a tener en cuenta en la creación de entornos de aprendizaje más eficientes y satisfactorios para el usuario.

La consecuente personalización del aprendizaje ofrecida en base a características del estudiante, como nivel de cono- 
cimientos o estilos de aprendizaje o intereses del usuario, ha consistido en adaptar los contenidos o en adaptar la navegación. En Vélez, Baldiris, Nassiff \& Fabregat (2008) se recoge una extensa bibliografía al respecto.

Una vez establecidas las variables del modelo de usuario a considerar en la adaptación de la formación, el siguiente paso es decidir la herramienta tecnológica que permita su implementación. Así, por ejemplo, Vélez, Baldiris, Nassiff \& Fabregat (2008) explican la generación de dos cursos adaptativos basados en dos variables o características del usuario: el nivel de conocimientos y las metas del usuario. La recogida de esos datos del usuario se realiza en el LMS y para la generación de los cursos se usa el modelo de referencia SCORM y la especificación IMS-LD. La decisión de los autores de utilizar ambos métodos se justifica plenamente por la importancia de la interoperabilidad y reusabilidad que así quedan plenamente garantizadas.

Nuestra decisión fue distinta, optamos por usar un LMS como Moodle, con todas las herramientas y facilidades de gestión y seguimiento de estudiantes que proporciona. Queremos incidir en este punto, a nuestro juicio muy importante. Los desarrolladores de sistemas de aprendizaje adaptativos no pueden olvidar que el éxito de tales sistemas depende de que los formadores o expertos en diferentes disciplinas los utilicen. Para ello creemos que es necesario que los entornos sean amigables y fáciles de usar, de forma que no se requieran habilidades de programación ni excesivo esfuerzo de implementación, y que tengan funcionalidades que permitan a los tutores hacer un seguimiento fácil del progreso de los estudiantes. En resumen, nos gustaría que los LMS avanzaran con paso firme en la línea de dotarlos de avanzadas funcionalidades de personalización del aprendizaje facilitando, además, el trabajo integral del profesor, como diseñador, tutor, evaluador, moderador, etc.

\section{EXPERIENCIAS SOBRE APRENDIZAJE ADAPTATIVO}

Nuestra experiencia en el ámbito de la personalización del aprendizaje se ha apoyado en el uso de Moodle con las funcionalidades de adaptatividad desarrolladas como complemento a la instalación estándar. Del uso inicial del módulo Activity Locking pasamos al uso de la herramienta de adaptatividad más potente que conocemos en un LMS: los condicionales del CICEI para Moodle.

El módulo Activity Locking para la versión 1.8 de Moodle, más que personalizar el aprendizaje, permitía adaptar la visualización y/o realización de las actividades del curso a las acciones del estudiante. En realidad, los contenidos son comunes a todos los estudiantes y únicamente algunos están bloqueados en tanto el estudiante no cumpla los requisitos exigidos en la configuración.

En el año 2008 se realizaron dos cursos en línea de apoyo a la docencia presencial de asignaturas que utilizan matemáticas en las titulaciones de Ingeniería e Ingeniería Técnica, entonces vigentes.

Los contenidos matemáticos de ambos cursos ya habian sido probados, en su versión italiana, por varias universidades del norte de Italia (CILEA, http://teorema.cilea.it) desde el año 2003. La instalación del módulo Activity Locking añadió a Moodle 1.8 la posibilidad de diseñar un aprendizaje más dirigido. El alumno avanzaba, en el acceso a las secciones de teoría y en la realización de los cuestionarios, dependiendo de su propio progreso. Las condiciones de avance se basaban, por ejemplo, en la puntuación obtenida en el test de ingreso o en el acceso a los recursos teóricos. Es decir, únicamente se adaptaba la visualización de los contenidos al ritmo de trabajo, pero todos los alumnos partieron del mismo punto y llegaron al final de las actividades, cubriendo todas ellas. Se trata más bien de dirigir al estudiante en su camino de aprendizaje de modo que realice las actividades en el orden previsto por el diseño de la instrucción.

En ambos cursos se dispuso de un test de evaluación inicial y otro final con el fin de registrar el avance en el nivel de conocimientos. También se registró información proporcionada por el estudiante en relación a la motivación extrínseca (utilidad e importancia de la materia), la motivación intrínseca (satisfacción por haber realizado el curso), la amigabilidad del sistema y la opinión sobre la metodología utilizada. Se constató un alto nivel de entusiasmo y una excelente opinión tanto del método como de las herramientas.

Posteriormente, la modalidad de personalización del aprendizaje que hemos trabajado es la de generar diseños 
de instrucción que se van adaptando a las características (datos del estudiante) y a las acciones del estudiante (datos de uso). Desde un punto de vista óptimo, la personalización de la educación exige que el profesor conozca al instante lo que un estudiante está aprendiendo, los errores que está cometiendo y que actúe de inmediato. Además, es importante que conozca su formación previa y sus circunstancias sociales, que adapte el recorrido educativo a su estilo de aprendizaje e incluso que conozca y utilice las emociones con las que cada estudiante aborda el aprendizaje.

Esa tarea es irrealizable en contextos en los que el profesor es responsable de organizar el aprendizaje de un elevado número de alumnos. Pero la viabilidad mejora cuando somos capaces de "clasificar" el tipo de personalización que pretendemos y limitamos y definimos las variables a considerar y esto se hace posible cuando se utiliza la tecnología para llevarlo a cabo.

Dos características tecnológicas han sido determinantes en la implementación de los diseños instruccionales adaptativos: los agrupamientos de Moodle, que permiten asignar recursos y actividades específicamente a un grupo de participantes, y los condicionales del CICEI para Moodle, que admiten una visualización cambiante del curso según el avance del estudiante y la asignación de recursos y actividades en función de sus características y actuación.

Nuestra propia experiencia nos ha enseñado que hay que avanzar paso a paso y asumiendo retos de creciente dificultad. El mirar nuestro proceso de trabajo nos permite proponer diferentes tipos de personalización basados en:

- Las credenciales del estudiante.

- Qué han aprendido o qué están aprendiendo.

- Cómo aprenden o cómo prefieren aprender.

- Las características emocionales.

El diseño basado en las credenciales del estudiante, como por ejemplo, sus acreditaciones académicas o perfil curricular, o bien su pertenencia a un grupo determinado, como un departamento concreto de una empresa o pertenecer a un intervalo de edad o estar matriculado en cierto curso de un grado determinado, etc. En definitiva, características personales debidas a su entorno laboral, social, académico, etc. que hacen presuponer unos conocimientos adquiridos, unos intereses determinados o unos medios específicos del grupo poblacional en el que está integrado cada participante.

Durante el primer trimestre de 2008 se ofreció, a los estudiantes matriculados en Matemáticas y Estadística de la Diplomatura en Ciencias Empresariales de la Universitat Jaume I de Castellón la realización de un curso de "Repaso de Matemáticas".

El público objetivo está, por tanto, segmentado inicialmente en los estudiantes de la asignatura de Matemáticas, por un lado, y los de Estadística, por otro. Esta división es la que se tuvo en cuenta para seleccionar qué contenidos eran mostrados a cada grupo de estudiantes. Es decir, la variable de adaptatividad considerada fue la de pertenencia a un grupo (Matemáticas o Estadistica), entendiendo que ello determinaba, a priori, las necesidades formativas de los estudiantes de ese grupo. En este caso usamos la versión 1.9 de Moodle que integra la funcionalidad agrupamientos, mediante la cual se pudo asignar contenidos diferenciados para cada agrupamiento.

En el diseño personalizado basado en qué han aprendido o qué están aprendiendo durante el proceso formativo son claves la evaluación diagnóstica inicial y las evaluaciones formativas intermedias que encaminan al estudiante hacia diferentes recursos y actividades según el aprendizaje demostrado en esas evaluaciones. En esta línea se diseñaron y experimentaron tres cursos adaptativos online en el año académico 2009-10:

- "Curso de Apoyo de Matemáticas", de octubre a diciembre de 2009 (CAM-2009).

- "Curso de repaso de Trigonometría", de marzo a abril de 2010 (TGM-2010).

- "Functions", mayo de 2010 (FNC-2010).

En los tres casos se ofreció al estudiante un complemento formativo en matemáticas para ayudarle en su proceso de aprendizaje en las titulaciones de Ingeniería y de Economía de diferentes universidades: CAM-2009 en la Universidad de Zaragoza y en la Universitat Jaume I de Castellón, TGM2010 en la Universidad de Zaragoza y, finalmente, FNC en la Technical University of Ostrava (República Checa). Cada uno de esos cursos abordaba los asuntos matemáticos más adecuados para cada contexto formativo. 
El diseño e implementación de los tres cursos adaptativos se realizó en Moodle 1.9 con los condicionales del CICEI. Aunque actualmente la versión 2.1 de Moodle ya permite cierto grado de adaptatividad, como ya hemos comentado, no es suficiente para los propósitos de dichos cursos. En

En el caso de CAM-2009, las variables de personalización consideradas se encuadran en las características del usuario descritas en la sección anterior. Se trata de cuatro variables sobre el nivel de conocimiento mostrado por el estudiante respecto a cuatro asuntos matemáticos. Se realizó una evaluación diagnóstica que determinó cada nivel y, como consecuencia, el sistema ofreció diferentes presentaciones instruccionales adaptadas al nivel mostrado. En la figura 1 se aprecia el diseño adaptativo realizado. Los iconos en forma de rombo indican la localización de las condiciones de adaptación; los números 1 al 4 incluidos en los rombos de la derecha indican que se condicionaba la aparición de los recursos subsiguientes a las respuestas del estudiante en diferentes preguntas del cuestionario inicial. Se puede consultar el detalle del diseño, implementación y resultados en Castelló, Lerís, Martínez \&t Sein-Echaluce (2010).

El diseño de los cursos TGM-2010 y FNC-2010 añade variables de interacción con el sistema, que solemos denominar variables de progreso del estudiante. En ambos casos, se realizó una evaluación diagnóstica inicial en la que los errores en las respuestas a determinadas preguntas deter-

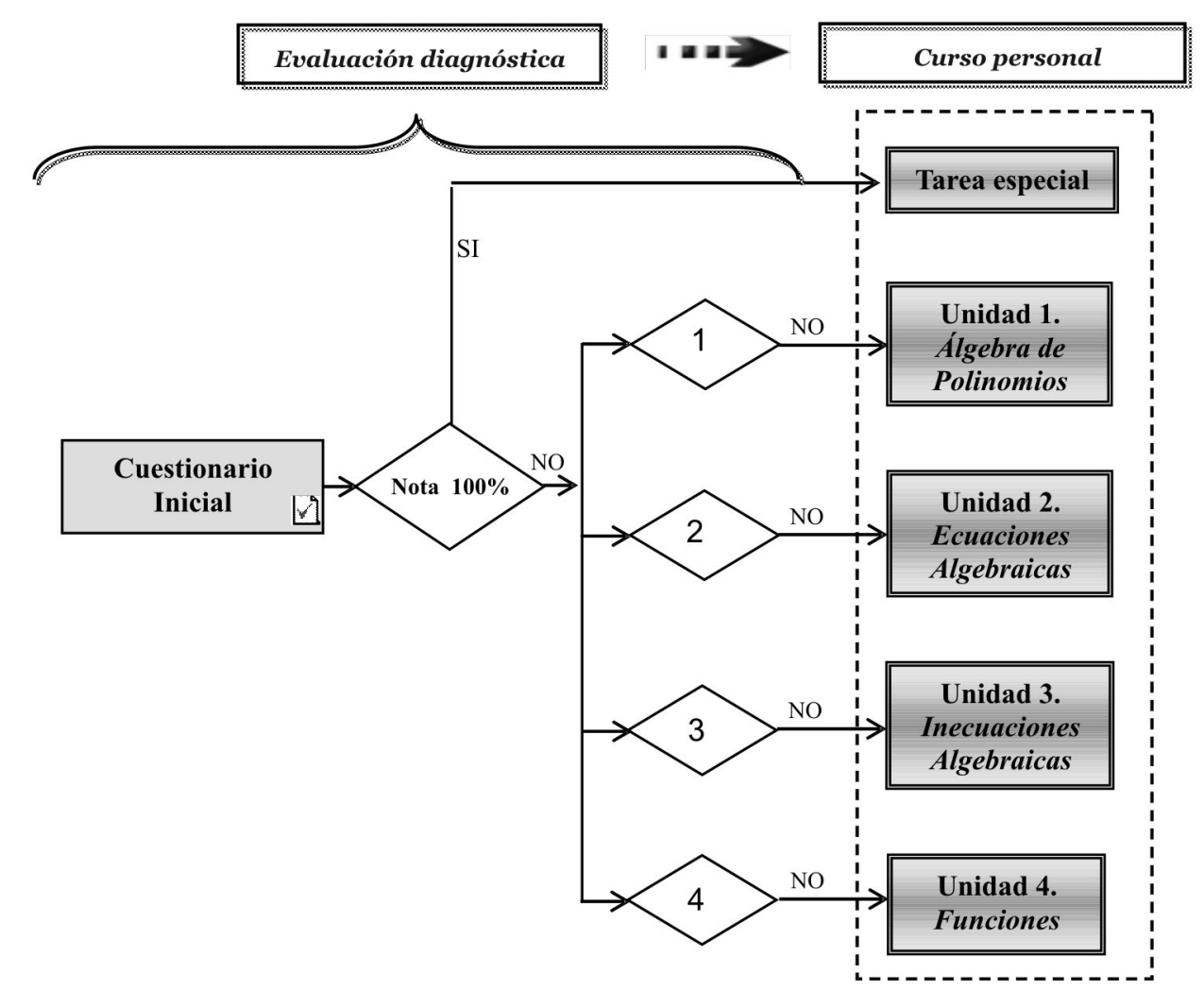

Figura 1. Esquema del diseño adaptativo de CAM-2009. 
minaban la aparición de unos u otros recursos. Además, el diseño instruccional marcó un conjunto de hitos, de puntos clave de aprendizaje, de modo que al evaluar las respuestas del estudiante a ciertas actividades el sistema le condujera a nuevos contenidos de refuerzo. El detalle del diseño y los resultados de su puesta en práctica están recogidos en Sein-Echaluce, Lerís \& Fidalgo (2011).

La figura 2 muestra una vista parcial del esquema de las condiciones o variables marcadas en el diseño instruccional del TGM-2010. Sin entrar en el detalle de cada recurso y de cada condición, esa figura 2 permite hacerse una idea del diseño a través de la forma de los iconos y sus colores. Así, los iconos con forma de rombo son los puntos en los que el sistema decide qué presentación mostrar al usuario en función de la acción realizada en los recursos previos. En la parte mostrada en la figura 2 hay tres condicionales, compuestos de varias condiciones enlazadas por conectores lógicos, que determinan la aparición de unos $u$ otros de los siguientes recursos.

Obsérvese que los rombos o las variables de adaptación se distribuyen a lo largo de la línea de avance en el curso (de izquierda a derecha en la figura 2). Los situados al principio forman parte de la evaluación diagnóstica (datos del usuario), en tanto que los situados más adelante constituyen las evaluaciones intermedias o de progreso del estudiante (datos de la acción).

En las tres experiencias se incluyó una evaluación final o prueba objetiva para medir el aprendizaje logrado $y_{\text {, }}$ además, se pidió al estudiante que expresara su opinión respondiendo a las preguntas de una encuesta publicada en el propio curso.

El tercer diseño instruccional aplicado, el basado en cómo aprenden o cómo prefieren aprender, se realizó en función de uno de los indicadores de personalización del aprendizaje más utilizado que es el estilo de aprendizaje predominante en cada estudiante.

En el curso académico 2010-11, se diseñó e implementó un curso online con cuatro diferentes diseños de instrucción, adaptados a cada uno de los cuatro estilos de aprendizaje de adultos que aparecen en el modelo de Honey-Munford (1986). El curso en línea fue diseñado para estudiantes de la asignatura Matemáticas II del primer curso de los nuevos grados de Ingeniería Informática y de Ingeniería Eléctrica de la Universidad de Zaragoza.

Como ya hemos dicho, el curso online contiene los diseños instruccionales de cuatro itinerarios, de modo que

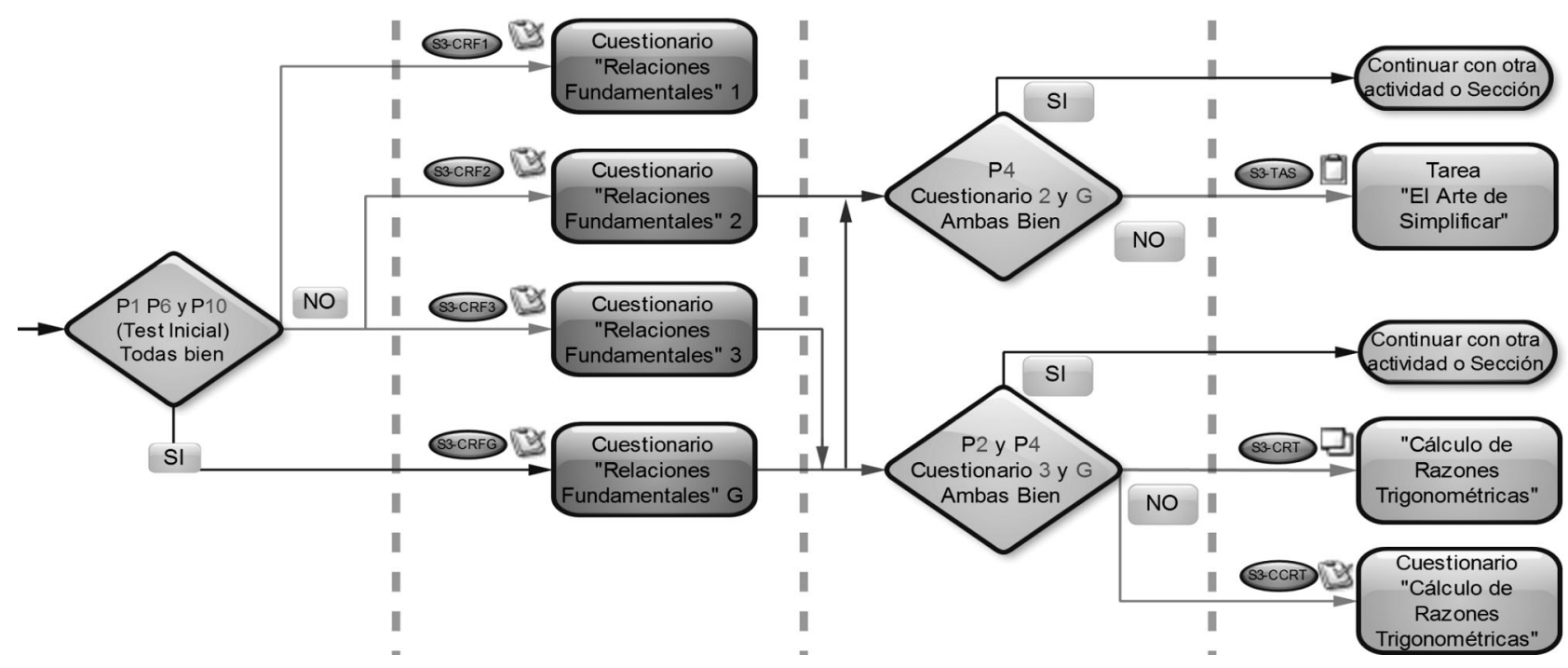

Figura 2. Vista parcial de la adaptación de la presentación a la acción del estudiante en el curso TGM-2010. 
cada estudiante realice el itinerario adaptado a su estilo de aprendizaje predominante. Para su determinación se utilizó el Cuestionario de Honey-Alonso de Estilos de Aprendizaje (CHAEA) de nodo que, a partir de las respuestas del estudiante a las 80 preguntas del cuestionario, se obtiene el grado de pertenencia del encuestado a cada uno de los cuatro estilos de aprendizaje. Este cuestionario fue incluido como módulo adicional de Moodle, desarrollo realizado por el CICEl, que junto con los condicionales, por ellos creados, fueron la base tecnológica necesaria para la implementación de un curso adaptativo de estas características. En la figura 3 se muestra el esquema de su diseño adaptativo.

Por último, hemos tenido en cuenta las características emocionales con las que se aborda el aprendizaje. Por ejemplo, la actitud del estudiante hacia su aprendizaje, que puede ser consecuencia de su motivación, de la valoración de la instrucción, de las experiencias en el estudio de la disciplina o de su auto-concepto como aprendiz, o el nivel de autonomía con el que emprende un proceso formativo.

Parece fuera de toda duda que las emociones influyen decisivamente en el aprendizaje. Por ejemplo, Penacoba,
Ecija, Velasco y Cigaran (2011) afirman que "la disposición afectiva previa de los componentes de un grupo afecta a la adquisición de la competencia genérica de trabajo en equipo". En consecuencia, el modelo de usuario, al que hemos hecho referencia en la sección anterior, debería contemplar las características emocionales del estudiante.

Claro está que decidir qué variables o rasgos son los más importantes, cómo medirlos y cómo actuar en consecuencia es especialmente relevante. Como aproximación a este asunto digamos que Wikipedia recoge la siguiente idea: "La combinación del Cociente Intelectual $(\mathrm{Cl})$ y el Cociente Emocional (CE), es la idea básica de la denominada Psicología positiva respecto al aprendizaje. En ella se establece que la motivación tiene un carácter emocional. Por ello el equilibrio emocional incrementa el aprendizaje." (extraído el 9 de noviembre de 2011). Parece, pues, que ambos cocientes podrian utilizarse como un indicador de adaptación de la instrucción.

En nuestro caso, el camino que hemos recorrido en esta línea se centra en qué variables medir y cómo medirlas. En septiembre de 2004 y 2006, se diseñó e implementó un cuestionario que evaluaba, entre otras, las siguien-

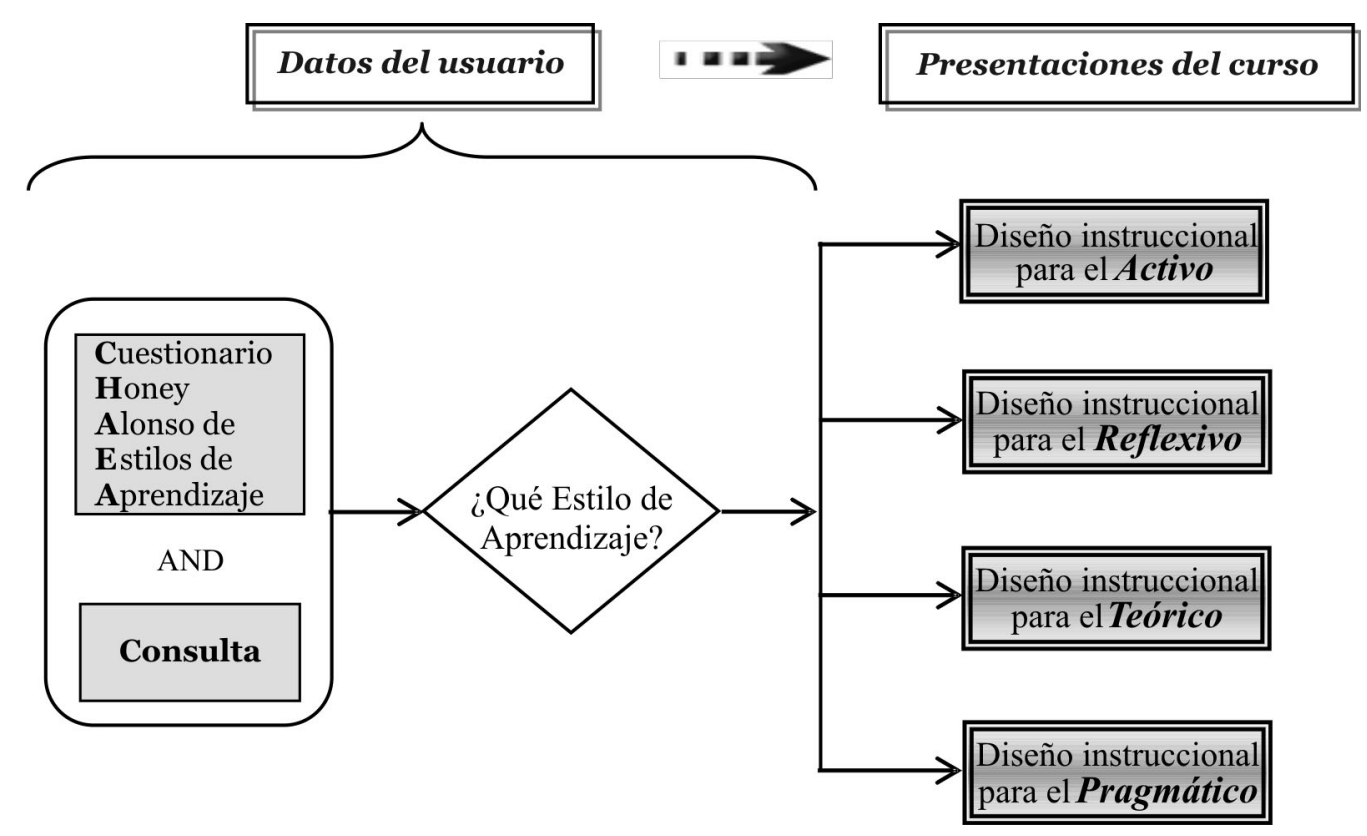

Figura 3. Diseño adaptativo basado en el estilo de aprendizaje. 
tes características del estudiante: actitud ante su propia formación y su perfil como aprendiz en el momento de iniciar sus estudios universitarios. Estas son las que ahora queremos destacar como relevantes para determinar las características emocionales del usuario. Decidimos fijar y medir el perfil de actitud del usuario hacia la formación que va a recibir a través de preguntas sobre: motivación, vocación o preferencia por los estudios que inicia, opinión sobre la formación matemática recibida, sentimientos hacia las matemáticas y su auto-concepto como estudiante de matemáticas. En cuanto al perfil del aprendiz, las preguntas se centraron en conocer ciertos aspectos en relación con sus costumbres, hábitos y habilidades al trabajar como aprendiz. En concreto, medimos tres aspectos del usuario: su independencia o autonomía para organizar su aprendizaje, su habilidad para el uso de la tecnología y su experiencia en el trabajo en equipo. Puede consultarse el detalle de estas evaluaciones diagnósticas y los resultados obtenidos en Bueno, Leris, Boal, Castelló, Correas, Martínez, Sein-Echaluce (2008).

Un siguiente paso sería establecer qué diseños instruccionales serian más acordes para adaptarse a las carac- terísticas emocionales del usuario. Como muestra de lo que se está haciendo, mencionamos el trabajo de Barrios \& González-Bravo (2011) en el que describen el Método de Aprendizaje Emocional que ellos aplican en el grado de Arquitectura.

Como resumen, en la figura 4 se observan las variables del modelo de usuario consideradas en nuestras experiencias. Las sombreadas en amarillo dieron lugar a cursos adaptativos diseñados, implementados, experimentados y evaluados; en tanto que la sombreada en naranja sólo ha sido tratada a nivel de diagnóstico, sin implementación en un curso adaptativo.

\section{Conclusiones}

En las secciones previas se presenta nuestra visión de la adaptabilidad o personalización del aprendizaje, desde las ideas ampliamente estudiadas y publicadas, hasta nuestra experiencia personal, nuestro conocimiento sobre el asunto.

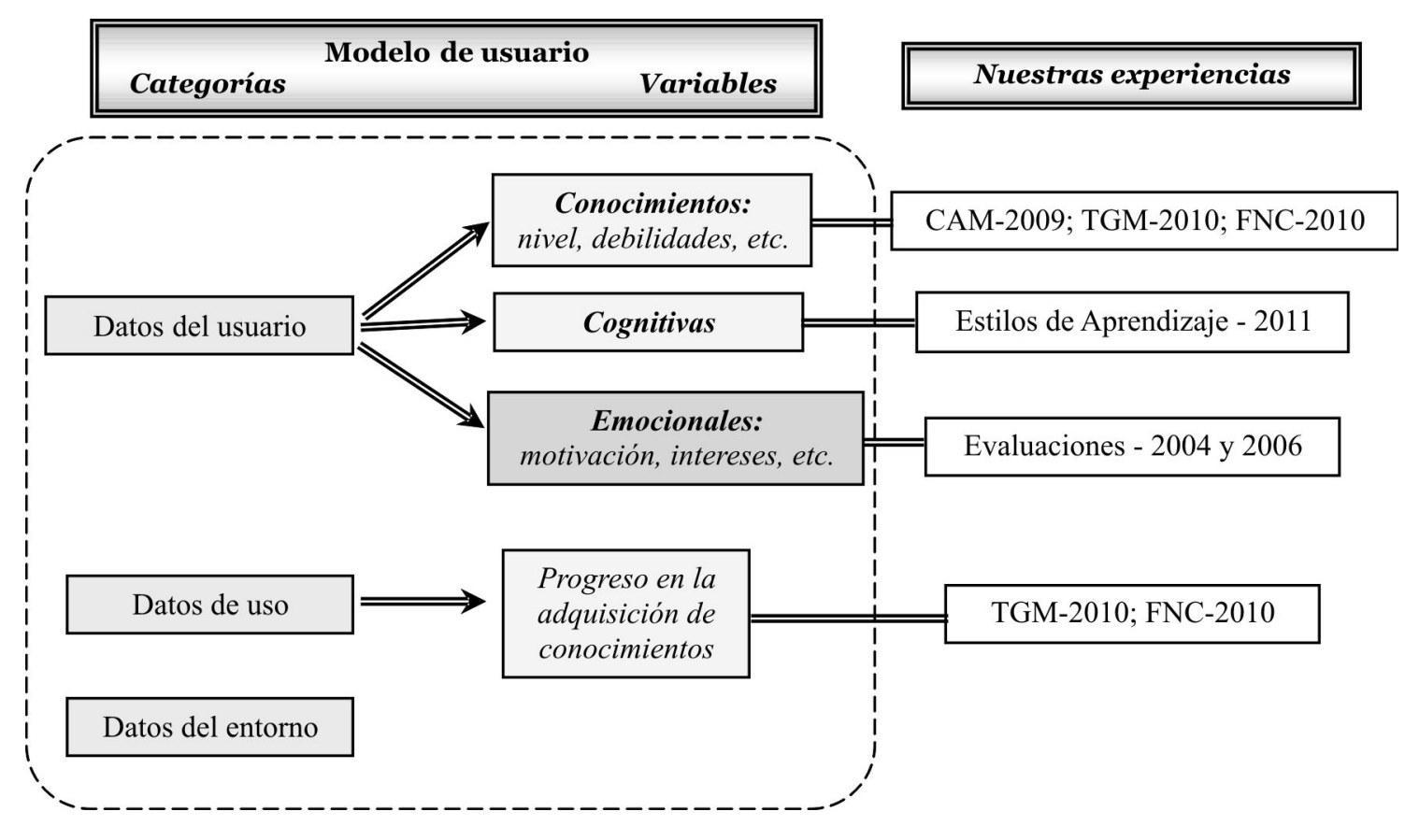

Figura 4. Esquema de variables de adaptación utilizadas en nuestras experiencias. 
El propósito ha sido mostrar, muy esquemáticamente, nuestro camino en la experimentación sobre el aprendizaje personalizado. En cualquier caso, estas experiencias suponen un necesario apoyo sobre el que sustentar lo que nos parece el principal objetivo: combinar todas estas facetas de la personalización, desde la grupal hasta la individual, y teniendo en cuenta desde los conocimientos y la forma en que aprendemos hasta las emociones.

Como ya hemos manifestado anteriormente, nuestra experiencia se circunscribe a la generación de diseños instruccionales evolutivos y adaptados a determinados rasgos del estudiante. En estos diseños el profesor actúa creando y organizando objetos de aprendizaje en forma de diferentes itinerarios y en función de una cantidad moderada de variables formativas. La consecuencia es un número fijo, más o menos elevado, de diseños instruccionales que van emergiendo según sean las acciones del estudiante y es la plataforma de e-learning, Moodle en nuestro caso, la que va seleccionando de forma automática los diferentes itinerarios de aprendizaje, previamente diseñados por el profesor, en función de las características y acciones del estudiante.

En el proceso de creación de los materiales de aprendizaje durante las experiencias realizadas, apreciamos la conveniencia de producir pequeños objetos evolutivos de aprendizaje con el fin de que admitieran diferentes presentaciones como actividades de Moodle y diferentes ubicaciones en el proceso de aprendizaje. Pero la gestión de objetos precisa el uso de repositorios de contenidos, de creación propia o de uso compartido, adecuadamente etiquetados. Estos repositorios, junto con sus buscadores o sistemas recomendadores, proporcionan la información solicitada por el usuario a través de formularios cruzados.

En este sentido, en Fidalgo, Balbín, Lerís \& Sein-Echaluce (2011) se explica el proceso de construcción y uso de un repositorio que gestiona los recursos seleccionados a partir del análisis de las mejores prácticas en la formación en competencias en los grados de Ingeniería. En el artículo se indican las dos partes claves de ese repositorio y buscador: el análisis de casos prácticos y el sistema de gestión de conocimiento. Respecto al primero se dice: "El objetivo del análisis consistió en identificar casos prácticos para cada competencia y sistematizarlos, con el fin de obtener criterios de clasificación y búsqueda". En cuanto al segun- do bloque, se dice: "El objetivo del sistema de gestión de conocimiento consistió en la clasificación, organización y desarrollo de un sistema de búsqueda de mejores prácticas aplicadas en la formación universitaria. El sistema de búsqueda, además de las búsquedas textuales (autor, universidad, palabras clave.....) y por bases de datos, permite la búsqueda a partir de competencias, metodologías, técnicas, recursos y herramientas."

Pero la producción de objetos de aprendizaje es un proceso costoso, por tanto es conveniente compartirlos (cederlos y utilizar los de otros) y en este sentido apostamos por el uso de las redes sociales y la gestión del conocimiento en abierto, lo que permite elegir los recursos más adecuados al modelo de usuario y mejorar el diseño personalizado del aprendizaje.

Lo anterior nos lleva a afirmar que el futuro está en los diseños personalizados del aprendizaje creados a partir del análisis de los objetos de aprendizaje, acorde con el contexto de utilización, así como la necesidad de utilizar un repositorio para su gestión, ya sea de los objetos de creación propia como del fruto de la cooperación en redes sociales y que permita las búsquedas a través de multietiquetas. Un objetivo a desarrollar es que los objetos elegidos, tras una búsqueda en el repositorio, puedan ser maquetados a gusto del usuario e incluso que se le ofrezcan guías para su organización según diferentes métodos o propósitos. Su aplicación permitiria al usuario disponer de todos los platos de un menú formativo de su gusto.

\section{Agradecimientos}

Este trabajo ha sido posible gracias a la Universidad de Zaragoza, el Gobierno de Aragón y el Fondo Social Europeo. La participación de Joaquín Castelló y Vicente Martínez de la Universitat Jaume I de Castellón, junto con el apoyo de esa institución, han hecho posible la realización de algunas de las experiencias expuestas. Así mismo, queremos agradecer a Enrique Rubio del CICEI (Universidad de Las Palmas de Gran canaria) su generosa disposición para utilizar sus desarrollos de los Condicionales en Moodle en los propios servidores del CICEl y a Ángel Fidalgo del LITI (Universidad Politécnica de Madrid) por iniciarnos en este apasionante tema y por sus valiosos y atinados consejos. 
Barrios, L. \&t González-Bravo, C. (2011): "Aproximación a una Metodología de Aprendizaje Emocional (MAE) en las enseñanzas de Arquitectura". Actas del I Congreso Internacional son Aprendizaje, Innovación y Competitividad (CINAIC 2011). Ed. Universidad Politécnica de Madrid. ISBN: 978-8496398-50-4.

Brusilovsky, P. (1996): Methods and Techniques of Adaptive Hypermedia: User Modeling and User-Adapted Interaction, Kluwer Academic Publishers, Vol. 6, pp. 87-129.

Brusilovsky, P. (2001): Adaptive Hypermedia, User Modeling and User-Adapted Interaction 11:87-110, 2001. 87. Kluwer Academic Publishers. Printed in the Netherlands.

Bueno, C.; Leris, D.; Boal, N.; Castelló, J.; Correas, J. M.; Martínez, V. \&t SeinEchaluce, M. L. (2008): Evaluation of attitudes and mathematical skills of first year university students. INTED2008 Proceedings International Technology, Education and Development. Publisher: IATED, Valencia, Spain. ISBN: 978-84-612-0190-7.

Castelló, J.; Lerís, D.; Martínez, V. \& SeinEchaluce, M. L. (2010): Personalized Learning on the Moodle Platform using the CICEI Conditionals: Support Course in Mathematics INTED2010 Proceedings International Technology, Education and Development. Publisher: IATED, Valencia, Spain. ISBN: 978-84-613-5538-9.

CICEI (2005): Centro de Innovación para la Sociedad de la Información. Universidad de Las Palmas de Gran Canaria, España. Implementación de condicionales http://www.cicei.com/index. php?option=com_content\&task=view ctid=288ctltemid=129 (consultado el 27 de noviembre de 2011)
Colón, L. (2011): Diseño instruccional, estrategias. http://www.slideshare.net/ Lynes/diseo-instruccional-estrategias (consultado el 27 de noviembre de 2011).

Fidalgo, A.; Balbín, A.; Lerís, D. \& SeinEchaluce, M. L. (2011): Repositorio de mejores prácticas aplicadas en la educación superior en ingeniería. Promotion and Innovation with New Technologies in Engineering Education (FINTDI). Digital Object Identifier: 10.1109/FINTDI.2011.5945969 Publication Year: 2011, Page(s): 1-7. IEEE Conferences.

Honey, P. S. \& Mumford, A. (1986): Using your learning styles. Maidenhead: Peter Honey.

Olea, J. \&t Ponsoda, V. (2002) Test adaptativos informatizados. Disponible en http://www.aristidesvara.net/prb/ metodologia/psicometria/teoria_respuesta/tai_aristidesvara.pdf (consultado el 27 de noviembre de 2011).

Penacoba, C.; Ecija, C.; Velasco, L. \& Cigaran, M. (2011): El afecto como variable relevante en la adquisición de la competencia de trabajo en equipo. Un programa de innovación en estudiantes de Ciencias de la Salud. Actas del I Congreso Internacional son Aprendizaje, Innovación y Competitividad (CINAIC 2011). Ed. Universidad Politécnica de Madrid. ISBN: 978-8496398-50-4.

Schooling for tomorrow- Personalising Education - ISBN-92-64-036598 ๔ OCDE 2006

Sein-Echaluce, M. L.; Lerís, D. \& Fidalgo, A. (2011): Diseño instruccional adaptativo de cursos online en Ingeniería. Promotion and Innovation with New Technologies in Engineering Education (FINTDI). Digital Object Identifier: $10.1109 /$ FINTDI.2011.5945972 Publication Year: 2011, Page(s): 1-8. IEEE Conferences. 
Vélez, J.; Baldiris, S.; Nassiff, S. \&t Fabregat, R. (2008): Generación de cursos virtuales adaptativos basados en SCORM e IMS-LD. Avances en Sistemas e Informática 5. 49-59.

Vélez, J. \&t Fabregat, R. (2007): Arquitectura para la Integración de las. Dimensiones de Adaptación en un Sistema Hipermedia Adaptativo. Published at Procedings of Research report Institut d'informatica i aplicacions (IIIA 07-01-RR).

Viñes, J. M. (2008): Diseño instruccional online (consultado el 27 de noviembre de 2011) http://www.aulatika. es/publicaciones/Diseno_instruccional_online.pdf

Williams, P.; Schrum, L.; Guardia, A. \&t Sangra, L. (2004): Modelos de diseño instruccional. In A. Sangra \&t L. Guardia (Eds.), Fundamentos del diseño instruccional con e-learning. Barcelona: Universitat Oberta de Cataluña. (consultado el 27 de noviembre de 2011) http://aulavirtualkamn.wikispaces.com/file/view/2.+MODELOS+DE+ DISE\%C3\%910+INSTRUCCIONAL.pdf 